\title{
Telehealth Technology: Potentials, Challenges and Research Directions for Developing Countries
}

\author{
M. Raza ${ }^{1}$, M. Hoa Le ${ }^{1}$, N. Aslam ${ }^{1}$, C. Hieu Le ${ }^{2}$, N. Tam Le ${ }^{3}$ and T. Ly Le \\ ${ }^{1}$ Faculty of Engineering and Environment, Northumbria University, Newcastle upon Tyne, United Kingdom \\ ${ }^{2}$ Faculty of Engineering and Science, University of Greenwich, Kent, United Kingdom \\ ${ }^{3}$ IBM Cloud, IBM Vietnam, Hanoi, Vietnam \\ ${ }^{4}$ School of Biotechnology, International University, Ho Chi Minh, Vietnam
}

\begin{abstract}
Telehealth has been developed and successfully applied in clinical practices, gained a strong interest and demonstrated its usefulness for medical diagnosis, treatments and rehabilitation worldwide. The advent of high speed communication technology and complex signal processing techniques, and recent advancements in cloud and cognitive computing, has created a new wave of opportunities for delivering remote healthcare applications and services, where the costeffective diagnosis and treatment solutions as well as healthcare services are important and need to be deployed widely. Nevertheless, there is still a significant challenge in fully adopting this technology due to asymmetry among the healthcare centers, hospitals and the user-ends, especially in developing countries. This paper provides an overview of the telehealth, then to addresses the possible telehealth technologies and applications that could be applied to improve the healthcare service performance, with the focus on the developing countries. The incorporation of different technologies in telehealth including, Internet of Things (IoT), cloud and cognitive computing, medical image processing and effective encoding is introduced and discussed. Finally, the possible research directions, challenges for the efficient telehealth, and potential research and technology collaborations are outlined.

Keywords - Telehealth, Internet of Things, Video Encoding, Medical technology
\end{abstract}

\section{INTRODUCTION}

Telehealth technology provides assistance for healthcare services that connect the inhabitants of rural areas to the provision of acceptable health care facilities. In the existing healthcare infrastructures, a conventional process for medical check-up is illustrated in Fig. 1. In this process, a patient needs to visit a clinic where the front desk registration and collection of vital signs (blood pressure, temperature etc.) takes place. Afterwards, the patient is assigned a time slot for doctor or specialist inspection, which results in medical prescription (including medicines, laboratory tests, revisits etc.) or further examinations. The patient's medical records are also updated to the service databank. Nevertheless, the availability of qualified doctors/specialists and basic medical equipment is limited in rural areas of developing countries and likely one has to travel long distance to get proper medical examination. This gap can be overcome with the assistance of information and communications technologies
(ICT). Hence, suitable health care facilities can be provisioned at the patient's doorstep thanks to cost-efficient portable medical equipment and reliable communications infrastructure [1-3]. In a typical telehealth-based health care system, medical check-up procedure is almost similar to the conventional check-up process but instead of travelling hundreds of miles to see a doctor in person, a patient can communicate with doctor via video conferencing and internet enabled medical equipment. Such a typical telehealth based examination procedure is depicted in Fig. 2.

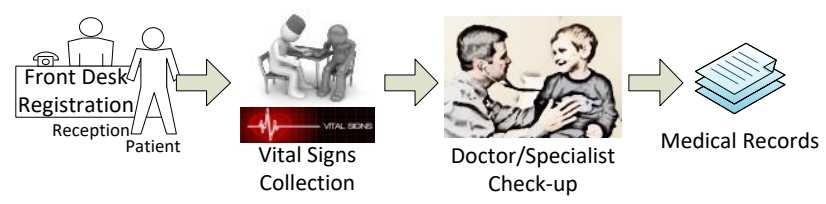

Fig. 1 Convention medical check-up procedure

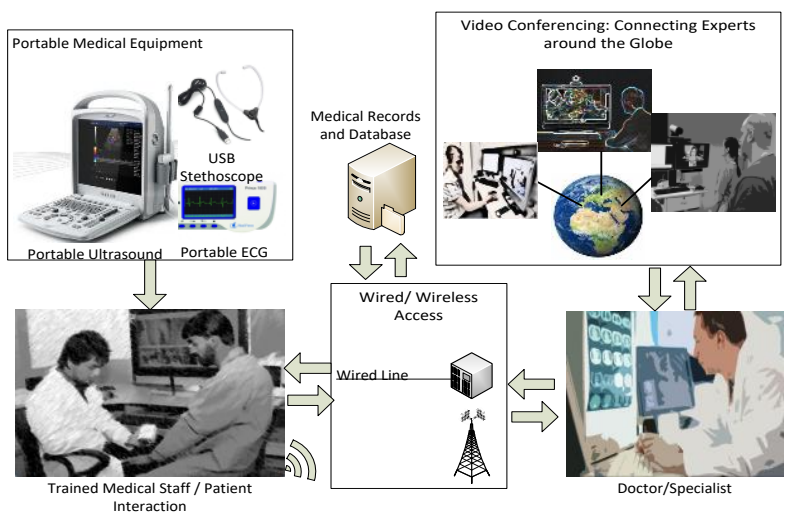

Fig. 2 Telehealth system $[7,8]$

Telehealth offers great benefits and in many cases ICT can serve to bridge the locality gaps. ICT also bears the potential to promptly link medical specialists all over the world to offer valuable expertise and necessary assistance in health care and medication [4]. Despite the great potential of the telehealth technology, lack of progress in its rapid adoption is mainly due to: (1) poor communication infrastructure, (2) lack of resources in developing countries, (3) lack of collaboration among health care organizations and (4) lack of government level initiative [5]. None the less, the experts are still hopeful that the adoption of telehealth in the 
developing countries can be great improvement in bridging the existing gaps in health care sectors [6]. Telehealth is a technology driven solution, which requires a basic communication infrastructure to link the remote areas with the metropolitan hospitals and medical facilities. However, the existence of obsolete communication technologies limits the use of telemedicine in the developing countries. Moreover, lack of research and development centers force the imports of portable medical equipment at relatively higher prices. This paper presents a review of telehealth technology, its limitations, and various challenges posed by ICT infrastructure in developing countries. In this paper, a solutionoriented approach is adopted, where tools and techniques are discussed to relax the constraints in most viable manner.

The rest of the paper is organized as follows: Health care facilities and role of telehealth in developing countries are presented in Section II. Section III discusses the limitations in Telehealth whereas section IV presents solutions for telehealth limitations and challenges. Finally, Section V concludes the discussion and gives final remarks.

\section{Health CARE IN DEVELOPING COUNTRIES AND ROLE OF TELEHEALTH}

Developing countries face multiple challenges that affect the health care provision. This includes lack of medical facilities, low doctor to patient ratios, insufficient funds, lack of awareness, poverty and unsafe water. According to the World Health Organization (WHO), respiratory diseases, malaria, unsafe water and unintentional poisoning kill 4.855 million people every year [9]. All these factors make health care provision very difficult especially with limited funds and resources. Under such circumstances, it is important to interfuse technology in health care sector to reap full benefits even with the limited resources. Telehealth technology has a foreseeable future as the low cost and efficient healthcare alternate solution for rising health problems. It is noticed that over 1 billion of world population is suffering from obesity giving rise to type 2 diabetes, high blood pressure, fatty liver disease, certain cancer types, strokes and heart diseases [10-12]. In addition, over 600 million people suffer from chronic diseases costing way over $\$ 500$ billion per year on health care [12] and a large portion of population is in need of assistive technologies due to cognitive diseases, invisible wounds, mental health and aging factors.

For all these problems telehealth can serve as a future technology, which offers a better fit for developing country financial resources. However, outdated ICT infrastructure limits the scope. It is therefore important to mold the research to offer solutions that can relax the constraints imposed by existing ICT. Details of mobile and broadband infrastructure are listed in Table 1, whereas the typical requirement for communications of various medical equip- ment and observatory investigation for telehealth services are summarized in Table 2 .

Table 1 ICT infrastructure internet and mobile $[13,14]$

\begin{tabular}{lcccc}
\hline Details & D -C & UD-C & World & LDC/RA \\
\hline Internet access & $81.3 \%$ & $34.1 \%$ & 46.4 & $6.7 \%$ \\
Internet Usage & $82.2 \%$ & $35.3 \%$ & $46.4 \%$ & $9.5 \%$ \\
Mobile broadband & $86.7 \%$ & $39.1 \%$ & $46.1 \%$ & $12.1 \%$ \\
3G Penetration & $50.04 \%$ & - & - & - \\
4G Penetration & $13.66 \%$ & - & - & - \\
\hline \multicolumn{4}{c}{ D-C: Developed Countries } & UD-C: Under developed countries \\
\multicolumn{2}{l}{ LDC/RA: Low developed countries /Rural Areas }
\end{tabular}

Table 2 Examples of medical application, communication requirements, and technologies [15-18]

\begin{tabular}{|c|c|c|c|c|c|c|}
\hline \multicolumn{4}{|c|}{ Data rate $\&$ accuracy requirements } & \multicolumn{3}{|c|}{ Technologies } \\
\hline Application & Data rate & Delay & BER & & $2 \mathrm{G}$ & $3 G$ \\
\hline $\mathrm{ECG}^{1}$ & $192 \mathrm{Kbps}$ & $\begin{array}{l}<250 \\
\mathrm{~ms}\end{array}$ & $<10^{-10}$ & BW & $64 \mathrm{Kbps}$ & $2 \mathrm{Mbps}$ \\
\hline $\mathrm{EEG}^{2}$ & $86.4 \mathrm{Kbps}$ & $\begin{array}{l}<250 \\
\mathrm{~ms}\end{array}$ & $<10^{-10}$ & $\mathrm{~T}$ & Digital & $\begin{array}{l}\text { CDMA } \\
2000\end{array}$ \\
\hline $\mathrm{EMG}^{3}$ & $\begin{array}{l}1.536 \\
\text { Mbps }\end{array}$ & $\begin{array}{l}<250 \\
\mathrm{~ms}\end{array}$ & $<10^{-10}$ & $S$ & $\begin{array}{l}\text { Packetized } \\
\text { Data }\end{array}$ & $\begin{array}{l}\text { Video / } \\
\text { data }\end{array}$ \\
\hline Drug delivery & $<16 \mathrm{bps}$ & $\begin{array}{l}<250 \\
\mathrm{~ms}\end{array}$ & $<10^{-10}$ & M & TDMA & CDMA \\
\hline $\begin{array}{l}\text { Glucose } \\
\text { monitoring }\end{array}$ & $<1 \mathrm{Kbps}$ & $\begin{array}{l}<250 \\
\mathrm{~ms}\end{array}$ & $<10^{-10}$ & Sw & $\begin{array}{l}\text { Circuit / } \\
\text { Packet }\end{array}$ & Packet \\
\hline Temperature & $<12$ bps & $<1 \mathrm{~s}$ & - & $\mathrm{CN}$ & PSTN & $\begin{array}{l}\text { Packet } \\
\text { Network }\end{array}$ \\
\hline Motion sensor & $<100 \mathrm{bps}$ & $\begin{array}{l}<300 \\
\mathrm{~ms}\end{array}$ & - & & & \\
\hline Audio stream & $1 \mathrm{Mbps}$ & $<20 \mathrm{~ms}$ & $<10^{-5}$ & & & \\
\hline Voice & $\begin{array}{l}50-100 \\
\text { Kbps }\end{array}$ & $\begin{array}{l}<100 \\
\mathrm{~ms}\end{array}$ & $<10^{-3}$ & & & \\
\hline $\begin{array}{l}\text { ECG: Electroc } \\
\text { EEG: Electroe } \\
\text { EMG: Electror }\end{array}$ & $\begin{array}{l}\text { ardiogram } \\
\text { acephalogran } \\
\text { nyogram }\end{array}$ & & $\begin{array}{l}\text { W: Band } \\
\text { Techno } \\
\text { Service }\end{array}$ & & $\begin{array}{l}\text { M: } M u l \\
\text { Sw: } S w \\
\text { CN: } C o\end{array}$ & $\begin{array}{l}\text { plexing } \\
\text { ching } \\
\text { Network }\end{array}$ \\
\hline
\end{tabular}

\section{Telehealth ChaLLENGES IN DEVELOPING COUNTRIES}

\section{A. Asymmetrical telehealth infrastructure}

One of the significant issues appeared in telehealth is the asymmetrical infrastructure of the communication systems where the doctor's end located in urban areas has high speed internet connection whereas the patient's end, mostly residing in a rural area, usually has much slower speed connection. Further-more, the infrastructure in the rural areas in developing countries utilizes obsolete technologies where data speed in the uplinks is much slower than downlinks. This issue is not good for telehealth, unfortunately, as the majority of transmitted information is from patient to doctor, thus a slow uplink creates a bottleneck especially in case of ultrasound videos and video conferencing.

\section{B. Integration of medical equipments with internet}

Another main issue in telehealth is the non-availability of Internet protocol (IP) enabled medical equipment to communicate the ongoing observations (Ultrasound, ECG etc.) 
from patient to the doctor/specialist, separated by a large distance. Most of the developing countries import medical equip-ment and hence a rapid transformation from legacy medical equipment to IP enabled need resources and time.

\section{Database formation}

In most of the cases, the patients' records are not centralized and in most of the developing countries, the patient's records are still maintained using traditional files. Though the use of telehealth offers a chance to establish a digital database for patients, it also presents a major challenge to develop a centralized database to keep track and access patients' health status. Furthermore, the access to the database and log maintenance requires extensive training programs for the doctors and medical staff.

\section{Privacy and secure communication}

The patient's privacy is a matter of utmost importance. The use of telehealth system can be exploited for hacking to the patient database [19]. Most of the communication taking place in the legacy networks is vulnerable to external threats and attacks and in case of disclosure could lead to greater influence on individual's life. Apart from these, appointment management system, user-friendly interface development, training of medical staff, information analysis and resource allocation and portability of medical equipment are also some of the challenges telehealth faces.

\section{E. Telehealth infrastructure development, benchmarking and information distribution}

For any telehealth system, it is very important that a development in one part of the world can be integrated in an overall stack of telehealth and telemedicine. Unfortunately, lack of benchmarking in this field has served a major bottleneck for the evolution of this technology and resisted the development of sound telehealth infrastructure.

\section{Health Care solutions and Feasibility OF TELEHEALTH IN DEVELOPING COUNTRIES}

The following are the outlines of techniques and possible research directions to overcome the above mentioned challenges about telehealth, especially for developing countries.

\section{A. Telehealth driven bit allocation, rate control scheme and adaptive image encoding}

In most of the medical diagnosis cases, Region of Interest (RoI) in a whole frame is very small and it needs identifying. For instance, if a doctor is interested in observing a wound on a patient, then rest of the graphic information captured by a camera including the surroundings of that wound would not be relevant, and they should not be transmitted in high resolution or in some cases can be neglected at all. It is therefore important to embed image processing, segmentation, RoI enhancement and pattern identification in telehealth systems for selective encoding in captured video frames. This technique, along with video compression offers highly reduced communication bandwidth requirements even be met by a low speed communication infrastructure (Table 1). In video communication, correlation based interframe identifiers can locate stationery areas in consecutive frames and hence, can devise a suitable bit allocation and rate control scheme. Moreover, adaptive encoding can be used to counter the bandwidth fluctuations in ICT by changing video resolution accordingly.

\section{$B$. Video encoding and compression}

It is not feasible to change the communication infrastructure in developing countries at once but one can easily identify the need of alternate solutions to assist telehealth systems over existing low speed asymmetrical communication infrastructure. Video encoding and compression (H.264/H.265 based background omitted encoding with interactive RoI) offers a significant reduction in bandwidth requirements. Much research [20-22] can be witnessed in this domain still there is much room for improvement.

\section{Medical equipments and Internet of Things (IoT)}

IoT is a rapidly emerging technology that can provide an interface for the communication of information generated by medical equipments over internet [23]. This technology makes a step forward for legacy telehealth systems as no new equipment is required for medical assistance in telehealth systems. Rather, the existing equipment available in remote places can be transformed into IP-enabled devices for which products like UART/SPI (WHZ9189) and others can be readily used.

\section{Computer based diagnosis}

Telehealth systems give a platform for evidence and symptoms based disease diagnosis to improve the authenticity of doctor's prescription and to reduce time per patient with automated and computer based opinion. However, for such systems to be existent researchers have to offer solutions for data analysis and diagnosis, based on probabilistic analysis, Bayesian decision theory and ensemble classifiers and recognition systems.

\section{E. Information based preventive measures}

Another off the shelf benefit of this technology is to provide the governing bodies with enough information to take preventive measures to avoid wide spread of viral and communicable diseases. A well-established telehealth infrastructure can be used to analyze region wise disease patterns and formulate action plan for the problems at hand.

\section{iv. Conclusions}

Telehealth has been developed and successfully applied in clinical practices, gained a strong interest and demon- 
strated its usefulness for medical diagnosis, treatments and rehabilitation worldwide. The advent of high speed communication technology and complex signal processing techniques, and recent advancements in cloud and cognitive computing, has created a new wave of opportunities for delivering remote healthcare applications and services, where the cost-effective diagnosis and treatment solutions as well as healthcare services are important and need to be deployed widely. Moreover, with the use of smart sensors and lab-on-chips technologies, it is possible to develop telehealth infrastructure to provide the cost-effective medical services, leading to the positive impacts on the lives of poor populations and directly contributing to the economic development and social welfare of developing countries.

Especially, IBM launched IBM Watson Health in April 2015. With the investment of more than $\$ 4$ billion, Watson Health has become one of the world's most diverse and largest assembly of health-related data [24]. This opens opportunities to create innovative and cost-effective telehealth solutions, specifically to promote the emerging value-based care model that aims at improving quality care and near- and long-term health outcomes for individuals while controlling costs. The roles of telehealth in healthcare, with the strong capabilities of cloud and cognitive computing, are now becoming more and more important. Recently, Bumrungrad International Hospital in Thailand has recruited IBM Watson Health to improve the quality of cancer care; the system will help doctors use medical evidence, published research and each patient's profile to plan the most effective treatments for cancer patients. IBM Vietnam is planning to collaborate with the local hospitals and research institutions to develop telehealth applications based on IBM Watson Health. This is potential for the research institutions, especially in UK and Vietnam, to collaboratively work on the cost-effective and innovative solutions to improve the quality of diagnosis and treatments, particularly to improve the health outcomes of underserved populations who are inadequately provided with a healthcare service or facility, and who are not able to access to the good healthcare services.

\section{ACKNOWLEDGMENT}

Erasmus Mundus cLINK programme and British Council - Newton Fund are acknowledged for support, funding, and facilitating the collaboration.

\section{REFERENCES}

1. C. Min et al. (2013) Enabling comfortable sports therapy for patient: A novel lightweight durable and portable ECG monitoring system. In e-Health Networking, Applications \& Services; IEEE 15th International Conference, 2013, pp 271-273
2. T. H. Tsai et al. (2012) Low-Power Analog Integrated Circuits for Wireless ECG Acquisition Systems. IEEE Transactions on Information Technology in Biomedicine; 16: 907-917

3. E. Ahmed and R. Kohno (2013) Medical reliable network using concatenated channel codes through GSM network. Engineering in Medicine and Biology Society (EMBC), 35th Annual International Conference of the IEEE; 2013, pp 4755-4758

4. J. Nyamu et al. (2015) An empirical study of collaborative innovation as a facilitator to telemedicine adoption in developing countries. International Conference on Information Society (i-Society 2015), 2015, pp 33-38

5. Telemedicine-Opportunities and developments in Member States. WHO, Global Observatory for eHealth, 2010.

6. R. S. Weinstein (2010) History of Telemedicine. Evolution, Context, and Transformation. Journal of Telemedicine and Telecare; 16: 167168.

7. V. A. S. N. H. C. System Telehealth - VA Sierra Nevada Health Care System: www.reno.va.gov [Access: April 2016]

8. Plastic Stethoscope Transcription Headset with Inline Volume Control and USB plug: ww.transcriptiongear.com [Access: April 2016]

9. Priority environment and health risks: www.who.int [Access: April 2016]

10. R. Trobec et al. (2011) Telehealth: A myth or reality? MIPRO, 2011 Proceedings of the 34th International Convention; 2011, pp. 295-300.

11. Overweight and Obesity Statistics: www.niddk.nih.gov [Access: April 2016]

12. Health and Wellness "statistics and insights". Continua Health Alliance: www.continuaalliance.org [Access: April 2016]

13. ICTFacts \& Figures: www.itu.int [Access: April 2016]

14. Global mobile statistics 2014: www.mobiforge.com [Access: April 2016]

15. B. Latré et al. (2011) A survey on wireless body area networks. Wireless Networks; 17: 1-18

16. R. Cavallari et al. (2014) A survey on wireless body area networks: technologies and design challenges. Communications Surveys \& Tutorials, IEEE; 16: 1635-1657

17. F. Touati and R. Tabish (2013) U-healthcare system: State-of-the-art review and challenges.Journal of medical systems; 37: 1-20

18. P. Sharma (2013) Evolution of mobile wireless communication networks-1G to $5 \mathrm{G}$ as well as future prospective of next generation communication network. International Journal of Computer Science and Mobile Computing; 2: 47-53

19. R. Trobec, U. Stani, and x010D, "Telehealth: A myth or reality?," in MIPRO, 2011 Proceedings of the 34th International Convention; 2011, pp. 295-300

20. A. Panayides et al. (2013) High-resolution, low-delay, and errorresilient medical ultrasound video communication using $\mathrm{H}$. 264/AVC over mobile WiMAX networks. Biomedical and Health Informatics, IEEE Journal of, 2013: 17, 619-628

21. C. N. Doukas et al. (2007) Advanced medical video services through context-aware medical networks. Engineering in Medicine and Biology Society. EMBS 2007, 29th Annual International Conference of the IEEE; 2007, pp 3074-3077

22. S. Khire et al. (2012) Region-of-interest video coding for enabling surgical telementoring in low-bandwidth scenarios," in Military Communications Conference, 2012-MILCOM, 2012, pp 1-6

23. F. Hu, D. Xie, and S. Shen (2013) On the application of the internet of things in the field of medical and health care. Green Computing and Communications. IEEE International Conference on and IEEE Cyber, Physical and Social Computing; 2013, pp 2053-2058

24. IDC link (2016) How Watson Health is circumventing the traditional information generation life cycle: www.idc.com [Access: April 2016]

Author: Mohsin Raza

Institute: Northumbria University

Street: Newcastle City Campus, 2 Ellison P1

City: Newcastle upon Tyne

Country: UK

Email: mohsin.raza@ northumbria.ac.uk 